01

\title{
Самовращение частиц с фотоиндуцируемым электрическим дипольным моментом
}

\author{
(C) А.И. Грачев \\ Физико-технический институт им. А.Ф. Иоффре, \\ 194021 Санкт-Петербург, Россия \\ e-mail: grach.shuv@mail.ioffe.ru
}

Поступило в Редакцию 9 марта 2019 г.

В окончательной редакции 3 февраля 2020 г.

Принято к публикации 5 марта 2020 г.

Обсуждается реализация вращения частиц с фотогенерируемым дипольным моментом в отсутствие внешнего источника поля. В основе обсуждаемых эффектов лежит механизм ориентационного взаимодействия, по крайней мере, пары частиц, являющихся фотодиполями. Рассмотрены примеры реализации указанного механизма, в том числе геометрия, отвечающая фотовращению Квинке. С практической точки зрения интерес может представлять разновидность самовращения в случае одиночного фотодиполя, находящегося на или вблизи металлической поверхности. Представлены оценки крутящего момента для сферических частиц с величиной радиуса $10 \mu \mathrm{m}$.

Ключевые слова: электрический дипольный момент, ориентационное взаимодействие, вращение, микро(нано)сфера.

DOI: $10.21883 / J T F .2020 .08 .49532 .87-19$

В работах $[1,2]$ впервые рассмотрено явления вращения в постоянном электрическом поле сферической частицы с фотогенерируемым в результате ее непрерывного освещения электрическим дипольным моментом. Геометрия экспериментов, описанных в $[1,2]$, отличается ориентацией индуцируемого диполя $\mathbf{P}_{p h}$ относительно вектора приложенного электрического поля $\mathbf{E}_{\mathrm{ex}}$. Если в [1] вектор $\mathbf{P}_{p h}$ ортогонален $\mathbf{E}_{\mathrm{ex}}$ и вращение частицы возможно при любом значении последнего, то в [2] векторы $\mathbf{P}_{p h}$ и $\mathbf{E}_{\mathrm{ex}}$ антипараллельны друг другу (геометрия вращения Квинке (ВК) [3,4]). В последнем случае вращение частицы, погруженной в вязкую среду, возможно лишь при превышении полем $E_{\mathrm{ex}}$ некоторого критического значения $E_{c}$ [4]. Однако, поскольку фотоиндуцированное вращения ВК может быть реализовано в любой газовой среде и даже в вакууме [2], величина $E_{c}$ может быть сведена практически к нулю.

Необходимость использования внешнего электрического поля может стать определенным препятствием в случае практической реализации эффекта фотоиндуцированного вращения. В настоящей работе обсуждается возможность вращения освещаемой сферической частицы (фотодиполя) в условиях отсутствия внешнего электрического поля, когда появление у нее крутящего момента обусловлено электростатическим (ориентационным) взаимодействием фотодиполя с, как минимум, еще одной частицей, обладающей дипольным моментом. Интересным вариантом реализации механизма самовращения является случай одиночного фотодиполя, расположенного вблизи или на металлической поверхности. Данный эффект объясняется взаимодействием фотодиполя с индуцируемым им зарядом поверхности и может стать основой для создания нового способа оптико-механической манипуляции микро- и наночастицами.

Явление диполь-дипольного взаимодействия частиц, обладающих постоянным электрическим (или магнитным) дипольным моментом, хорошо известно. В качестве примера на рис. 1 показано взаимодействие двух электрических диполей, центры которых находятся на расстоянии $r$ (которое следует рассматривать, как вектор r) друг от друга, а моменты $P_{1}$ и $P_{2}$ ориентированы параллельно. Электрическое поле каждого из них $\left(\mathbf{E}_{1}\right.$ и $\mathbf{E}_{2}$ соответственно) продуцирует крутящий момент соседнего диполя равный:

$$
\mathbf{T}_{1,2}=\mathbf{P}_{1,2} \times \mathbf{E}_{2,1}
$$

Если $P_{1}=P_{2}$, то диполи будут поворачиваться с одинаковой скоростью, стремясь сориентировать моменты вдоль $\mathbf{r}$, что в конечном итоге и произойдет. Подобное взаимодействие будет иметь место и в том случае, когда дипольные моменты $\mathbf{P}_{p h}^{(1)}$ и $\mathbf{P}_{p h}^{(2)}$ (рис. 1) генерируются в результате одного из рассмотренных в [1] фотоэффектов. Однако теперь ориентации $\mathbf{P}_{p h}^{(1)}$ и $\mathbf{P}_{p h}^{(2)}$ должны оставаться неизменными при повороте каждой из частиц, по крайней мере, в условиях их адиабатического вращения $[1,5]$, поэтому каждая из них будет испытывать постоянный крутящий момент, что должно привести к их непрерывному вращению. Динамика вращения сферических частиц в условиях реализации приближения точечных диполей $(r \gg R$, где $R$ - радиуса частиц) будет описываться следующим 


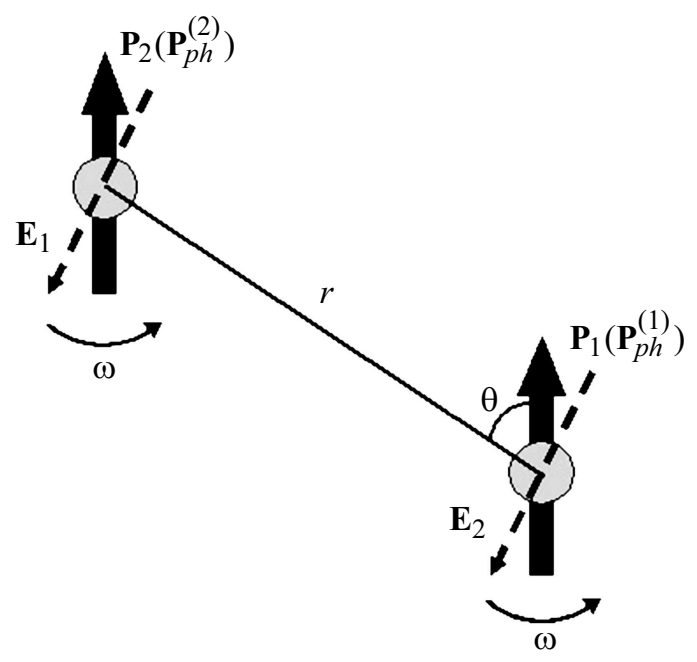

Рис. 1. Электростатическое взаимодействие точечных диполей (фотодиполей) с параллельно ориентированными дипольными моментами. Изогнутые стрелки указывают направление поворота (вращения) вектора дипольного момента.

уравнением [5]:

$$
J(d \omega(t) / d t)=3 P_{p h}^{2} \sin 2 \theta / 8 \pi \varepsilon_{0} \varepsilon_{m} r^{3}-\gamma \omega,
$$

где $\theta-$ угол между $r$ и $\mathbf{P}_{p h}^{(1)}\left(\mathbf{P}_{p h}^{(2)}\right), \varepsilon_{m}-$ диэлектрическая проницаемость жидкой (газовой) среды, в которой находятся частицы, а $\gamma$ - коэффициент трения вращения сферы в указанной среде. Как и в случае эффекта, рассмотренного в [1], стационарная скорость вращения частиц будет определяться условием равенства двух членов в правой части (2).

Использование уравнения (2) ограничено условием адиабатического вращения частицы [1,5], когда изменением ориентации дипольного момента вращающейся сферы можно пренебречь. При нарушении данного условия с ростом величины $\omega$ вектор эффективного дипольного момента каждой из сфер будет все больше отклоняться в сторону $\mathbf{r}$ [5]. В отличие от вращения во внешнем электрическом поле это будет сопровождаться и изменением направления вектора индуцируемого фотодиполем поля $\mathbf{E}_{p h}$, поэтому переход к режиму неадиабатического вращения приведет к появлению зависимости $\theta$ от $\omega$, в результате чего линейное уравнение (2) должно трансформироваться в нелинейное. Следует также учесть и уменьшение крутящего момента $T$ вследствие снижения эффективного дипольного момента вращающейся сферы в сравнении с $P_{p h}[5]$.

Описание движения частиц требует также учета их взаимной электрической поляризации, что особенно важно, если они находятся в среде с низкой вязкостью, например, в вакууме. Кроме того, следует помнить о трансляционном движении диполей, вызванном пространственно-неоднородным характером поля $\mathbf{E}_{p h}$. Так, в геометрии рис. 1 частицы должны перемещаться навстречу друг другу в направлении $\mathbf{r}$ вследствие дей- ствия силы $F_{r}$ :

$$
F_{r}=P_{p h}^{2}\left(1-3 \cos ^{2} \theta\right) / 8 \pi \varepsilon_{0} \varepsilon_{m} r^{4} .
$$

Поэтому в общем случае необходимо искать решение (2) совместно с уравнением, описывающим трансляционное движение диполей. Динамика частиц при учете их электрогидродинамического взаимодействия рассматривалась, например, в [6,7] для случая вращения Квинке. Аналогичная задача неизбежно возникнет и для системы фотодиполей как при наличии, так в отсутствие приложенного электрического поля.

Частным случаем взаимодействия фотодиполей является геометрия, в которой оба вектора, $\mathbf{P}_{p h}^{(1)}$ и $\mathbf{P}_{p h}^{(2)}$, ортогональны $r$. Хотя крутящий момент для каждой из частиц отсутствует, данная ориентация отвечает условию неустойчивого равновесия, когда случайное отклонение вектора момента одного из диполей может вызвать их совместное вращение. Нетрудно понять, что данная геометрия отвечает для каждого их них условиям наблюдения фотовращения Квинке [2]. Следует отметить, что в отличие от вышеописанной ориентации, где вектор $\omega$ ортогонален плоскости, в которой лежат оба вектора $\mathbf{P}_{p h}^{(1)}$ и $\mathbf{P}_{p h}^{(2)}$, в данной геометрии должно выполняться менее строгое условие: $\boldsymbol{\omega} \perp \mathbf{P}_{p h}^{(1)}\left(\mathbf{P}_{p h}^{(2)}\right) \quad$ (в случае ВК аналогичное условие выглядит, как $\left.\boldsymbol{\omega} \perp \mathbf{E}_{\mathrm{ex}}\right)$ и диполи неизбежно должны вращаться в противоположных направлениях $\left(\omega_{1}=-\omega_{2}\right)$. Поскольку на относительно больших расстояниях поле диполя не слишком велико, проявления данного варианта фотовращения Квинке следует ожидать лишь для близко расположенных частиц, находящихся в среде с малой вязкостью, например, в вакууме [2].

Выше предполагалось, что частицы помещены в жидкую или газообразную среду, однако с практической точки зрения интерес может представлять случай системы фотодиполей, находящихся на поверхности некоего материала. Подобные эксперименты могут оказаться полезными, например, при исследовании механизмов трения на микро- и наноуровне. Современные экспериментальные методы в области микро-трибологии базируются на технике атомно-силовой микроскопии $[8,9]$. Это позволяет изучать процессы трения скольжения и качения частиц, но практически исключает возможность исследования трения верчения при ортогональной ориентации крутящего момента относительно поверхности. Оптический способ задания направления $\mathbf{P}_{p h}$ позволяет легко изменять условия взаимодействия частиц и, как результат, осуществлять переход от одного типа их перемещения по поверхности к другому. Рис. 2 демонстрируют примеры ориентации векторов $\mathbf{P}_{p h}^{(1)}$ и $\mathbf{P}_{p h}^{(2)}$, отвечающие чистому качению или верчению сфер соответственно. Очевидно, что электрическое поле фотодиполей должно вести к появлению индуцированного заряда подложки и, следовательно, к необходимости учета его взаимодействия с каждым из диполей. В контексте данного замечания лежит идея реализации еще 


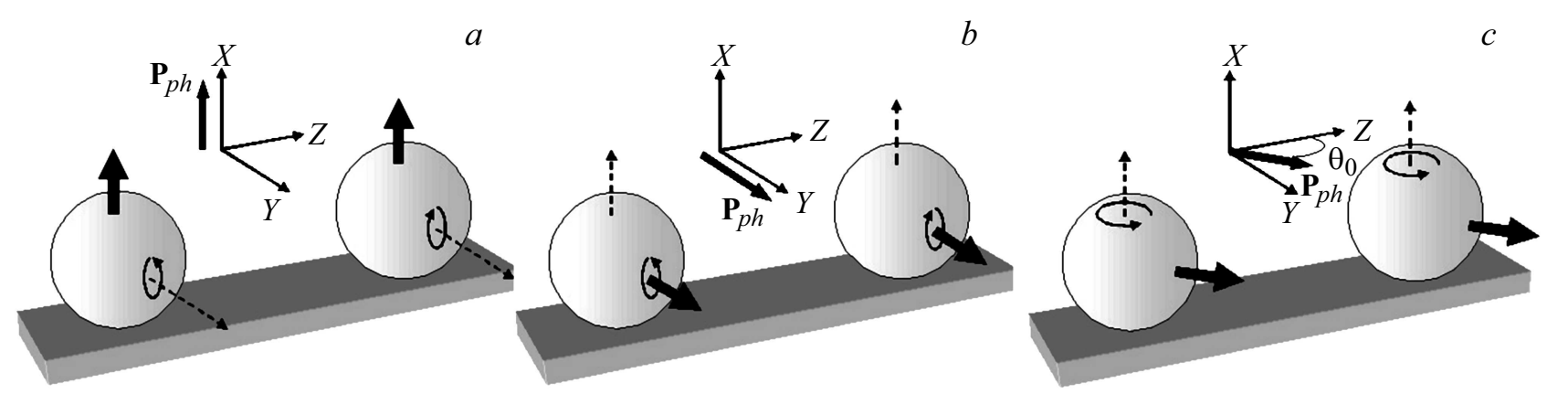

Рис. 2. Примеры взаимодействия пары сферических фотодиполей, находящихся на твердой поверхности, в том случае, когда векторы фотоиндуцируемого дипольного момента ортогональны поверхности $(a)$, параллельны поверхности и ортогональны линии, соединяющий центры сфер $(b)$, параллельны поверхности и образуют „магический угол“ $\theta_{0}=\arccos (1 / \sqrt{3}) \approx 54.7^{\circ}$ относительно линии, соединяющей центры сфер $(c)$.

одной разновидности самоиндуцированного вращения фотодиполя, на этот раз одиночного. Дело в том, что если вектор $\mathbf{P}_{p h}$ фотодиполя, помещенного вблизи или на плоской поверхности проводящей среды (рис. 3), образует относительно нормали к плоскости угол $\varphi$, отличный от 0 или $\pi / 2$, то электрическое взаимодействие диполя с индуцируемым им зарядом поверхности приведет к появлению вращающего момента $T_{i m}$. Величина $T_{i m}$ легко вычисляется (в случае бесконечной заземленной плоскости) с использованием метода изображений, как взаимодействие реального диполя и его отображения (рис. 3). Таким образом, теперь отпадает необходимость в наличии второго реального диполя для обеспечения крутящего момента сферы, способного вызвать ее движение по поверхности. Важным моментом является сравнительная простота перемещения сферы по поверхности в любом заданном направлении путем варьирования азимутального угла падающего светового пучка, в то время как изменение полярного угла $\varphi$ позволяет контролировать ее скорость.

Взаимодействие фотодиполей можно рассчитать, используя представленные в [1] оценки $P_{p h}$, точнее, генерируемого внутри частицы однородного электрического поля $\mathbf{E}_{p h}^{\text {int }}$ для каждого из представленных механизмов. Следует, однако, иметь в виду, что в [1] предполагалось выполненным условие равенства показателей преломления материала фотодиполя и окружающей его жидкой среды. Экспериментальная реализация данного условия достаточно проблематична в случае неорганических фотопроводников, но существенно упрощается для органических полупроводников (фотополимеров), величина показателя преломления большинства из которых находится в интервале 1.4-1.7 [10]. Однако, например, при трибологических исследованиях частицы могут находиться в воздушной (газовой) среде или в вакууме. В принципе, используя для освещения микролинзы, можно также обеспечить практически параллельное прохождение светового луча внутри сферической частицы. Для этого необходимо, чтобы параметр дифракции частицы удовлетворял приближению геометрической оптики [11].

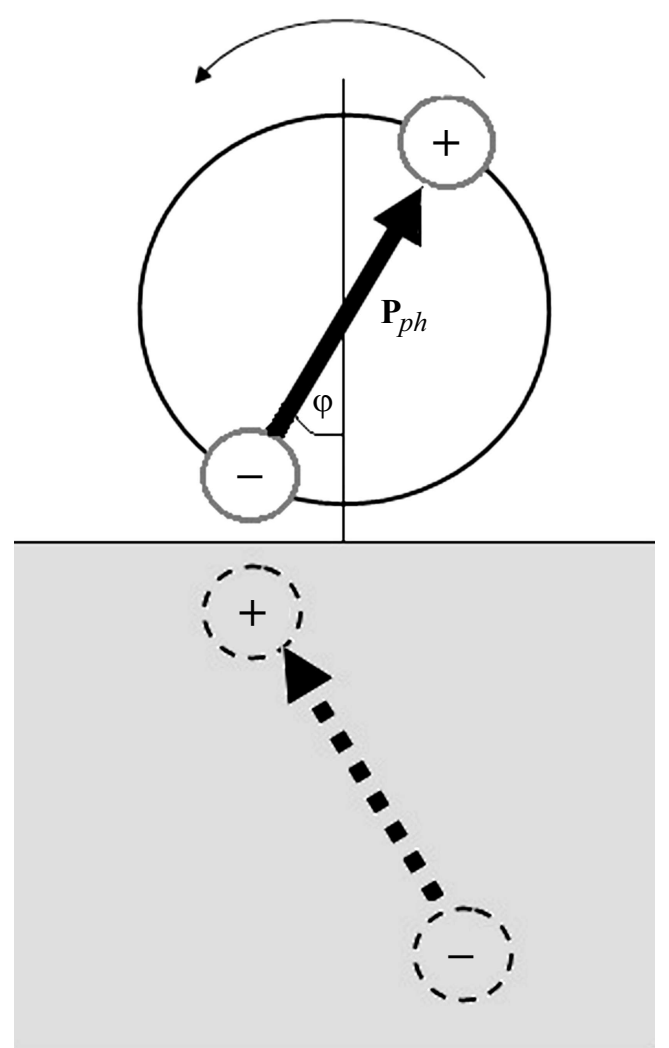

Рис. 3. Вращение фотодиполя, находящегося вблизи проводящей заземленной поверхности, обусловленное его взаимодействием с индуцируемым зарядом (фиктивным диполем), ведущим к появлению крутящего момента в том случае, когда угол $\varphi$ отличен от 0 или $\pi / 2$.

Таким образом, представленные в [1] расчеты следует рассматривать, как оценку сверху $E_{p h}^{i n t}$, учитывая, что при переходе в субмикронную область ее величина неизбежно будет снижаться (безотносительно к возрастающему влиянию поверхности). В целом же решение задачи о распределении световой мощности внутри сферической частицы при произвольных условиях ее освещения тре- 
бует использования численных методов расчета [11], что дополнительно осложняет определение фотопроводящих характеристик частицы [12], а следовательно, и $P_{p h}$.

Как демонстрируют недавно полученные экспериментальные данные [13] (фотогенерация $E_{p h}^{i n t} \geq 10^{4} \mathrm{~V} \cdot \mathrm{m}^{-1}$ в микрочастицах $\mathrm{Cu}_{2} \mathrm{O}$ ), сильное поглощение падающего света может снять в определенной степени проблему снижения Pрh в субмикронной области. В частности, это относится и к фотовольтаическому механизму генерации $P_{p h}[1]$, поэтому ниже будем предполагать именно его реализацию (отметим, что в данном контексте особый интерес может представлять прогрессирующая технология двухкомпонентных частиц типа ядро-оболочка). В качестве примера рассмотрим две находящиеся в вакууме частицы с $R=10 \mu \mathrm{m}$ в геометрии рис. 2, $a$, центры которых находятся на расстоянии $3 R$ друг от друга. Предполагая при этом справедливость (3) для $F_{r}$, играющей здесь роль силы тяги, получим следующую оценку:

$$
F_{r} \approx\left(6 \cdot 10^{2}\right) \varepsilon_{s} E_{p h}^{i n t} P_{p h} \approx 7.5 \cdot 10^{-11} \mathrm{~N}
$$

при $E_{p h}^{i n t}=10^{5} \mathrm{~V} \cdot \mathrm{m}^{-1}$ и $\varepsilon_{s}=10$. Величину $F_{r}$ следует сравнить с силами сопротивления качению сфер по поверхности, которые для частиц (суб)микронного размера определяются в первую очередь величиной межмолекулярного взаимодействия частица-поверхность (адгезией). Как показывает первичное сравнение представленных оценок с литературными данными, взаимодействие фотодиполей, по-видимому, способно обеспечить качение частиц (что важно, и их верчение), имеющих микронные размеры.

В заключение оценим крутящий момент $T$ частицы с теми же параметрами, которая левитирует в вакууме над проводящей поверхностью на расстоянии $d$, равном $2 R$ (от центра сферы). Используя известное выражение:

$$
T_{i m}=P_{p h}^{2} \sin 2 \varphi / 64 \pi \varepsilon_{0} d^{3}
$$

получим $T_{i m} \sim 10^{-15} \mathrm{~N} \cdot \mathrm{m}$ в случае $\varphi=45^{\circ}$.

Резюмируя, можно предположить, что рассмотренные эффекты самовращения фотодиполей позволят реализовать принципиально новые решения задачи создания систем оптико-механической манипуляции микрои наночастицами, не требующие приложения внешних электрических полей.

\section{Конфликт интересов}

Автор заявляет, что у него нет конфликта интересов.
[6] Dolinsky Yu., Elperin T. // Phys. Rev. E . 2012. Vol. 85. P. 026608.

[7] Das D., Saintillan D. // Phys. Rev. E. 2013. Vol. 87. P. 043014.

[8] Дедков Г.В. // УФН. 2000. Т. 170. С. 585.

[9] Sümer B., Sitti M.// J. Adhesion Sci. Technol. 2008. Vol. 22. P. 481.

[10] Brandrup J., Immergut E.H., Gruike E.A., Abe A., Bloch D.R. Polymer Handbook, 4th ed. NY:: John Wiley \& Sons, 2005.

[11] Liu L.H., Tan H.P., Tong T.W. // J. Quant. Spect. Radiat. Trans. 2002. Vol. 72. C. 747.

[12] Gharghi M., Bai H., Stevens G., Sivoththaman S. // IEEE Trans. Elec. Dev. 2006. Vol. 53. P. 1355.

[13] R. Chen, S. Pang, H. An, J. Zhu, S. Ye, Y. Gao, F. Fan, Can Li. Nat. Energy 3, 0194, (2018).

\section{Список литературы}

[1] Грачев А.И. // ФТТ. 2018. Т. 60. С. 666.

[2] Грачев А.И. // Письма в ЖТФ. 2018. Т. 44. С. 27.

[3] Quincke G. // Ann. Phys. Chem. Band. 1896. Vol. 59. C. 417.

[4] Jones T.B. // IEEE Trans. Ind. Appl. 1984. IA-20. C. 845.

[5] Грачев А.И. // ЖТФ. 2019. Т. 89. Вып. 1. С. 5. 